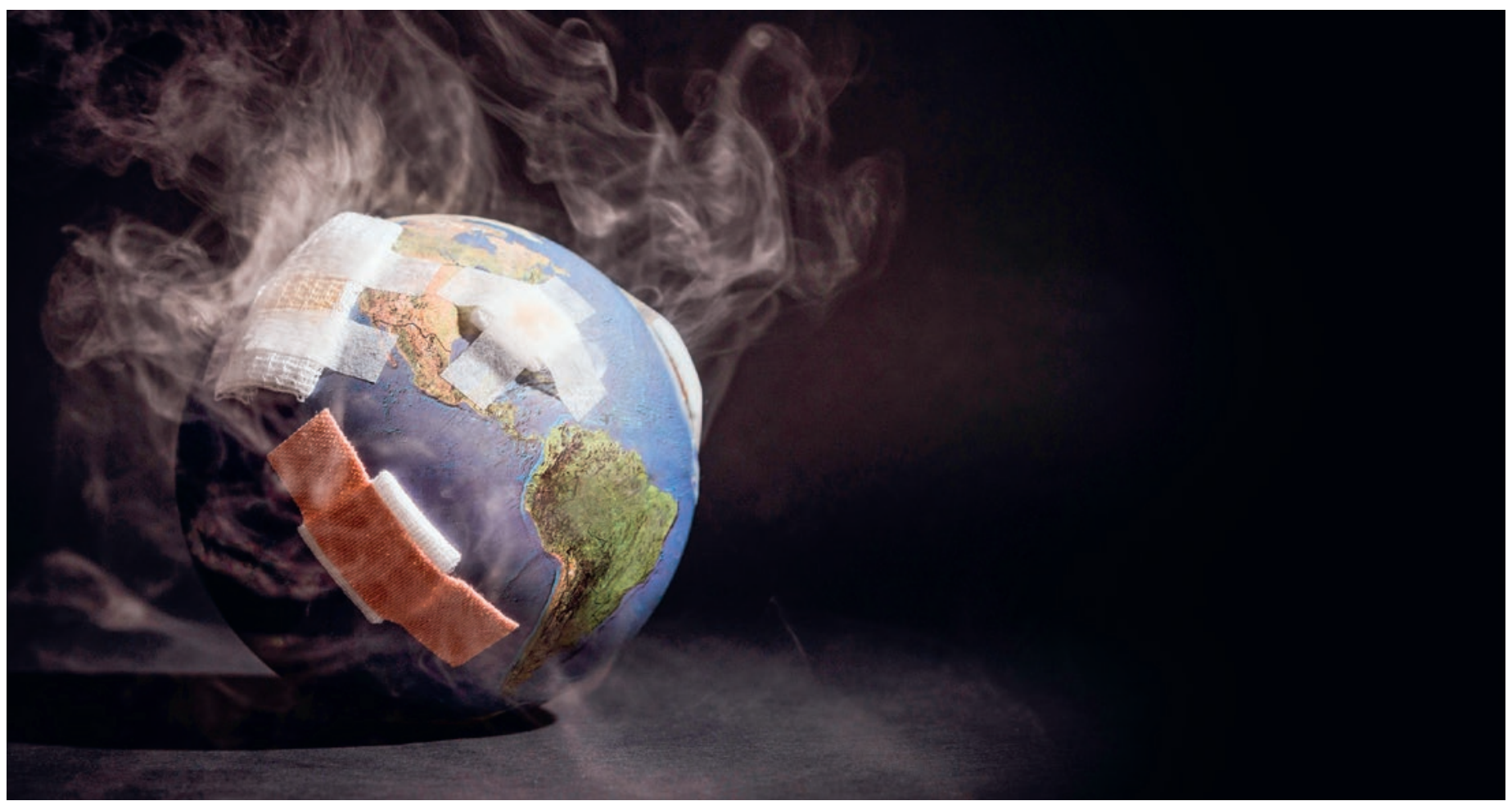

La terre en souffrance: les fonds des caisses de pension sont investis dans les énergies fossiles, néfastes pour le climat.

\title{
La catastrophe climatique et nos caisses de pension
}

René Jaccard ${ }^{a}$, Ueli Hagnauer ${ }^{b}$

a Dr méd., Commission scientifique Grands Parents pour le Climat, GPclimat.ch; ' Dr méd., Coordinateur Grands Parents pour le Climat, GPclimat.ch

Si l'on veut éviter que les efforts déployés pour se remettre des conséquences de la pandémie de Covid-19 ne conduisent à la prochaine catastrophe, celle de la crise climatique non maîtrisée, des critères de durabilité doivent déterminer le catalogue des mesures pandémiques et postpandémiques (mot-clé Green recovery) [3].

En pleine deuxième vague de la pandémie de coronavirus, la nouvelle tombe: le nombre de personnes qui meurent directement du réchauffement climatique et de l'utilisation de combustibles fossiles qui en découle continue d'augmenter d'année en année (en 2019: 7,5 millions au niveau mondial). Les maladies cardiaques, pulmonaires et rénales liées au climat sont en augmentation constante, et il en va de même pour les absences au travail dues au réchauffement climatique (en 2019: 302 milliards d'heures dans le monde) [1]. Parallèlement, les principaux paramètres climatiques ont continué à se détériorer: par rapport à 1950, la température annuelle a augmenté de $1,2^{\circ} \mathrm{C}$ dans le monde et de $1,7^{\circ} \mathrm{C}$ en Suisse. La concentration mondiale du principal gaz à effet de serre, le $\mathrm{CO}_{2}$, est passée de 250 à 410 ppm [2].

\section{Que pouvons-nous faire, nous les médecins?}

Les efforts pour mieux lutter contre les conséquences sanitaires des jours de forte chaleur ont déjà porté leurs fruits en Suisse [4]; des projets nationaux visant à recenser les émissions de gaz à effet de serre causées par le secteur des soins de santé ont été lancés, avec pour objectif déclaré de les réduire (mot-clé: Green Hospitals) [5]; les médecins en faveur de l'environnement (MfE) ont publié récemment un guide pour réduire l'empreinte de $\mathrm{CO}_{2}$ dans la pratique [6].

Nous aimerions ici discuter d'une autre possibilité ayant un potentiel d'impact particulièrement important: il s'agit de la manière dont les fonds des caisses de pension sont investies. 


\section{Placements cruciaux pour le climat}

Le montant total de l'épargne obligatoire des caisses de pension en Suisse est d'environ 1000 milliards de francs (l'équivalent d'environ $1 / 7$ du montant total de la place financière suisse). Les pratiques d'investissement actuelles des fonds de pension entraîneront une hausse de la température de 4 à $6^{\circ} \mathrm{C}$ d'ici la fin du siècle, notamment en raison des investissements élevés dans les énergies fossiles, dont le charbon, particulièrement nocif pour le climat et la santé [7]. Sur les 300000 personnes au moins qui travaillent dans le secteur de la santé en Suisse, certaines sont employées dans des institutions publiques et automatiquement affectées à des fonds correspondants. Cependant, une grande partie est assurée dans de petites entreprises, qui sont libres de choisir leur propre caisse de retraite.

Un classement de 110 caisses de pension suisses a récemment été publié [8]. Actuellement, seuls $8 \%$ des caisses ont un portefeuille axé sur la durabilité et la protection du climat. Parmi les moins bien classées (55\%!), on trouve malheureusement trois importantes caisses de pension principalement utilisées dans le secteur de la santé par le corps médical privé: les Fondations de prévoyance PAT-BVG, Pro Medico et ASMAC [8].

\section{Investissements en faveur de la transition énergétique}

Depuis 2008, un montant total de 42 milliards USD a été désinvesti par le secteur de la santé (en retirant les investissements dans les énergies fossiles). Y ont participé 23 organisations de médecins du Royaume-Uni, du Canada, d'Australie et d'Allemagne [1]. Il est difficile de comprendre pourquoi nos caisses de pension en Suisse, avec leur puissante organisation professionnelle FMH, ne s'engagent pas dans une politique d'investissement conforme aux objectifs climatiques de l'accord de Paris de 2015 (COP 21).

Aujourd'hui, continuer à investir dans les énergies fossiles ne fait pas qu'exacerber la crise climatique en cours et compromettre la santé des franges vulnérables de la population et des générations futures [1]. Cela signifie aussi que nous risquons de perdre des montants importants de notre épargne retraite. Outre la $\mathrm{FMH}$, nous pouvons toutes et tous contribuer à la représentation de nos intérêts vis-à-vis des caisses de pension: en nous référant au classement de notre caisse de pension en matière d'impact sur le climat et en demandant quelles sont les pistes d'amélioration. Si ces éléments ne nous satisfont pas, alors nous devons persister et signer: soit notre caisse s'engage à prendre un cap compatible avec la durabilité, soit nous nous tournons vers l'une des caisses de pension qui s'efforcent d'investir de manière respectueuse du climat (et qui, soit dit en passant, enregistrent d'excellents résultats). Après tout, la protection du climat n'est pas moins la promotion de la santé!

\section{Références}

1 Watts N, Amann M, Arnell N, et al. The 2020 report of the Lancet Countdown on health and climate change: responding to converging crises; publié en ligne le 2 décembre 2020: https://doi.org/10.1016/ S0140-6736(20)32290-X

2 OFEV, et al., 2020: Climate change in Switzerland. Indicateurs sur les causes, les impacts, les mesures. Etat de l'environnement $\mathrm{N}^{\circ} 2013,105$ pages.

3 UE, 3 juillet 2020: Boosting the EU's green recovery, en ligne: https://ec.europa.eu/commission/presscorner/detail/en/ IP_20_1250

4 Ragettli MS, Röösli M. Health effects of heat in Switzerland and the importance of prevention measures Swiss TPH, July 2020.

5 Muir K, Keller R, Stucki M: Bringing green best practice into hospitals with an LCA approach. 29 juillet 2020, Forum de discussion $\mathrm{N}^{\circ} 74$, IUNR et ZHAW.

6 AefU-Ratgeber Ökologie in der Arztpraxis, en ligne http://www. aefu.ch/fileadmin/user_upload/aefu-data/b_documents/themen/ praxisoekologie/Ratgeber_Energiecheck.pdf

7 BafU et PACTA, octobre 2017: Quantifying the alignment of Swiss pension funds and insurances with the Paris agreement, en ligne https://www.bafu.admin.ch/bafu/de/home/themen/klima/ fachinformationen/klima-und-finanzmarkt.html

8 Klima-Allianz Schweiz, 2 novembre 2020: Seule une fraction des fonds de pension investit de manière écologique. Online https:// www.klima-allianz.ch/klima-rating/?utm source $=$ Newsletter+Finanzwelt-Politik+DE+200702\&utm_campaign=b6ab87ae03-EMAIL_CAMPAIGN_2018_08_30_08_31_COPY 01\&utm_medium $=$ email\&utm term $=0$ f44f$\bar{f} 8 \mathrm{~b} 12 \mathrm{~b} 2-\mathrm{b} \overline{6} \mathrm{ab} 87 \mathrm{ae} 03-587539512$

\section{Crédits photo}

(c) Myboys.me | Dreamstime.com, image symbolique

\section{L'essentiel en bref}

- Le réchauffement climatique ne fait pas que menacer l'environnement, il entraîne une augmentation constante des maladies liées à ce changement. Protéger le climat revient donc à promouvoir la santé.

- Le placement des fonds des caisses de pension dans des portefeuilles axés sur la protection du climat a un grand potentiel d'impact: le montant total des avoirs de pension épargnés en Suisse représente $1 / 7$ du montant total de la place financière.

- Soit nos fonds d'épargne sont placés dans des secteurs durables, soit nous nous tournons vers l'une des caisses de pension qui misent sur des principes pro-climat. 